\title{
The Meissl scheme for the geodetic ellipsoid
}

\author{
S. J. Claessens ${ }^{1}$ corresponding author . \\ W. E. Featherstone ${ }^{2}$
}

12 November 2007

\begin{abstract}
We present a variant of the Meissl scheme to relate surface spherical harmonic coefficients of the disturbing potential of the Earth's gravity field on the surface of the geodetic ellipsoid to surface spherical harmonic coefficients of its first- and second-order normal derivatives on the same or any other ellipsoid. It extends the original [spherical] Meissl scheme, which only holds for harmonic coefficients computed from geodetic data on a sphere. In our scheme, a vector of solid spherical harmonic coefficients of one quantity is transformed into spherical harmonic coefficients of another quantity by pre-multiplication with a transformation matrix. This matrix is diagonal for transformations between spheres, but block-diagonal for transformations involving the ellipsoid. The computation of the transformation matrix involves an inversion if the original coefficients are defined on the ellipsoid. This inversion can be performed accurately and efficiently (i.e., without regularisation) for transformation among different gravity field quantities on the same ellipsoid, due to diagonal dominance of the matrices. However, transformations from the ellipsoid to another surface can only be performed accurately and efficiently for coefficients up to degree and order 520 due to numerical instabilities in the inversion.
\end{abstract}

Keywords Gravity field functionals - spectral theory geopotential models - spherical harmonics

Western Australian Centre for Geodesy \& The Institute for Geoscience Research, Curtin University of Technology, GPO Box U1987, Perth, WA 6845, Australia

1. E-mail: s.claessens@curtin.edu.au; tel: +61 892663505 ; fax: +61 8 92662703

2. E-mail: w.featherstone@ @urtin.edu.au; tel: +61 89266 2734; fax: +61892662703

\section{Introduction}

We provide some advance upon and further generalisation of the original spherical Meissl scheme (Meissl 1971, Rummel and Van Gelderen 1992, 1995, Grafarend 2001). Our main motivation is that much of current physical geodetic theory and practice is based on spherical approximations, whereas the oblate ellipsoid of revolution better describes the true figure of the Earth. As such, formulations that account for the Earth's ellipticity are preferable.

The relations among different gravity field quantities (functionals), e.g., the geopotential and its derivatives, are much simpler in the spectral domain than in the space domain (e.g., Heiskanen and Moritz 1967). These spectral relations can be combined in a 'diagram', often called the Meissl scheme. The principles of the spectral relations among gravity field functionals were formulated by Meissl (1971) and later combined into a concise scheme by Rummel and Van Gelderen (1992, 1995) and Grafarend (2001).

The main part of the spherical Meissl scheme relates surface spherical harmonic coefficients (SHCs) of the Earth's external disturbing potential $(T)$ with coefficients of its firstand second-order radial derivatives on spheres at different altitudes. The spherical Meissl scheme has been extended to include derivatives in latitudinal and longitudinal directions by using vector or tensor spherical harmonic expansions, instead of the common scalar spherical harmonic expansion (Rummel and Van Gelderen 1995). The spherical Meissl scheme was further extended to include vertical deflections and gravity disturbances (Grafarend 2001), and forward gravity field modelling using Newtonian integration (Kuhn and Featherstone 2003), while Keller (2002) discusses the Meissl scheme in terms of pseudo-differential operators.

However, all of the above contributions only apply to gravity field functionals given on a sphere. When the oblate ellipticity of the Earth $(e)$ is taken into account, a represen- 
tation of gravity quantities as a surface spherical harmonic expansion is still possible (cf. Claessens 2006). While spectral relations among them become more complicated, they exist nevertheless.

A second limitation of the original [spherical] Meissl scheme is that it only considers radial, longitudinal and latitudinal derivatives of $T$. Derivatives of $T$ with respect to the ellipsoidal surface normal $(h)$ are not included, even though these derivatives feature in many boundary-value problems (BVPs). A spectral relation between the disturbing potential and its first derivative with respect to $h$ are provided in Moritz (1980a, Ch. 39), and Sjöberg (2003), but these solutions are limited to an accuracy of order $\mathcal{O}\left(e^{2}\right)$, which is insufficient for high-degree coefficients. As an extension of the Meissl scheme to a non-spherical geometry, Bölling and Grafarend (2005) derive relations between $T$ and its derivatives in the ellipsoidal domain. They use the framework of ellipsoidal harmonics (e.g., Hobson 1931, Heiskanen and Moritz 1967, pp. 41-45) to derive the ellipsoidal spectral properties.

In this paper, the Meissl scheme is generalised to include relations among $T$ and its normal derivatives on the ellipsoid (i.e., with respect to the ellipsoidal surface normal $h$ ), in the framework of the conceptually simple and most commonly used scalar spherical harmonic expansion. A practical advantage of using scalar spherical harmonics, as opposed to tensor spherical harmonics and/or ellipsoidal harmonics, is that the vast majority of global gravity models are expressed in terms of scalar spherical harmonics. Synthesis of functionals from spherical harmonic expansions is straightforward and numerically stable up to very high degree and order (e.g., Holmes and Featherstone 2002, Jekeli et al. 2007, Wittwer et al. in press), whereas the numerical computation of ellipsoidal harmonics is more problematic (e.g., Sona 1995). Moreover, the use of the ellipsoidal coordinate system in combination with the more common geodetic coordinate system is not required in the approach taken here.

The scheme presented in this paper can be of use in many applications, such as the solution of ellipsoidal BVPs for gravity field modelling. A specific example is the construction of a global gravity field model from terrestrial data (cf., e.g., Cruz 1986; Petrovskaya et al. 2001; Claessens and Featherstone 2005). Terrestrial gravity anomalies can be expanded into a series of surface spherical harmonics on the ellipsoid and transformed into a solid spherical harmonic expansion, without the need for continuation to a bounding sphere as is required in the methods of Cruz (1986) and Petrovskaya et al. (2001). The second-order derivatives of the disturbing potential are of interest in gravity gradiometry, and the second-order derivative with respect to $h$ (i.e., the vertical gravity gradient) is, e.g., used in upward/down- ward continuation of gravity measurements (e.g., Heiskanen and Moritz 1967, p.114, Rózsa and Tóth 2005).

\section{A contextual recapitulation of the spherical Meissl scheme}

Any function that is harmonic outside a sphere with radius $R$, e.g., the Earth's exterior disturbing potential $T$, can be expanded into a series of solid spherical harmonics

$T(r, \theta, \lambda)=\sum_{n=0}^{\infty}\left(\frac{R}{r}\right)^{n+1} \sum_{m=-n}^{n} \bar{T}_{n m}^{R} \bar{Y}_{n m}(\theta, \lambda)$

where $\bar{Y}_{n m}$ are the fully-normalised spherical harmonic functions and the coefficients $\bar{T}_{n m}^{R}$ can be computed from an integration over the sphere (e.g., Heiskanen and Moritz 1967, Hobson 1931)

$\bar{T}_{n m}^{R}=\frac{1}{4 \pi} \int_{\sigma} T(R, \theta, \lambda) \bar{Y}_{n m}(\theta, \lambda) d \sigma$

where $(r, \theta, \lambda)$ are spherical polar coordinates and $d \sigma$ is the infinitesimal surface element on the unit sphere

$(d \sigma=\sin \theta d \theta d \lambda)$

Equation (1) can be used to compute $T$ and its radial derivatives anywhere outside the sphere that completely encloses the masses of the Earth. Simple one-to-one relations exist between these solid SHCs and surface SHCs of $T$ or its radial derivatives defined on the surface of a sphere situated completely within the harmonic region (Meissl 1971, Rummel and Van Gelderen 1992, 1995, Grafarend 2001).

These one-to-one [spherical] relations are all of the form (cf. Rummel and Van Gelderen 1995)

${\overline{d_{r}^{j}}}_{n m}^{R_{2}}=\lambda_{n}^{R R_{2}}\left(T, d_{r}^{j} T\right) \bar{T}_{n m}^{R}, \quad 0 \leq j \leq 2$

where ${\overline{d_{r}^{j}}}_{n m}^{R_{2}}$ are surface SHCs of the $j$-th radial derivative of $T$ on the surface of a sphere with radius $R_{2}$, and $\lambda_{n}^{R R_{2}}\left(T, d_{r}^{j} T\right)$ are the functions that transform the solid SHCs $\bar{T}_{n m}^{R}$ into the surface spherical harmonic coefficient ${\overline{d_{r}^{j}}}_{n m}^{R_{2}}$.

The transformation functions in Eq. (3) depend solely upon the degree $n$ of the coefficients and on the radii $R$ and $R_{2}$. Inserting the inverse of Eq. (3) (replacing index $j$ by $i$ ) into Eq. (3) itself, gives a more general relation among the functionals of $T$.

${\overline{d_{r}^{j} T}}_{n m}^{R_{2}}=\lambda_{n}^{R_{1} R_{2}}\left(d_{r}^{i} T, d_{r}^{j} T\right){\overline{d_{r}^{i} T}}_{n m}^{R_{1}}, \quad 0 \leq i, j \leq 2$

where

$\lambda_{n}^{R_{1} R_{2}}\left(d_{r}^{i} T, d_{r}^{j} T\right)=\frac{\lambda_{n}^{R R_{2}}\left(T, d_{r}^{j} T\right)}{\lambda_{n}^{R R_{1}}\left(T, d_{r}^{i} T\right)}$

It can be derived via evaluation and differentiation of Eq. (1) that

$\lambda_{n}^{R R_{1}}(T, T)=\left(\frac{R}{R_{1}}\right)^{n+1}$

$\lambda_{n}^{R R_{1}}\left(T, d_{r} T\right)=-\frac{n+1}{R}\left(\frac{R}{R_{1}}\right)^{n+2}$ 
$\lambda_{n}^{R R_{1}}\left(T, d_{r}^{2} T\right)=\frac{(n+1)(n+2)}{R^{2}}\left(\frac{R}{R_{1}}\right)^{n+3}$

Rummel and Van Gelderen (1995) propose a spherical Meissl scheme that relates $T$ to its first two radial derivatives on a sphere with radius $R$ ('ground level'), and to $T$ and its first two radial derivatives on a sphere with radius $R_{s}$ ('satellite height'). Using Eqs. (5-8) any transformation function $\lambda_{n}^{R_{1} R_{2}}\left(d_{r}^{i} T, d_{r}^{j} T\right)$ can be computed for $0 \leq i, j \leq$ 2. An adaptation, in the context of this paper, of this spherical Meissl scheme is shown in Fig. 1. The transformation functions $\lambda_{n}^{R_{1} R_{2}}\left(d_{r}^{i} T, d_{r}^{j} T\right)$ corresponding to the arrows in Fig. 1 can be viewed as eigenvalues. They are provided in Table 1 , where the subscripts $R_{1}$ and $R_{2}$ are shown only once when $R_{1}=R_{2}$.

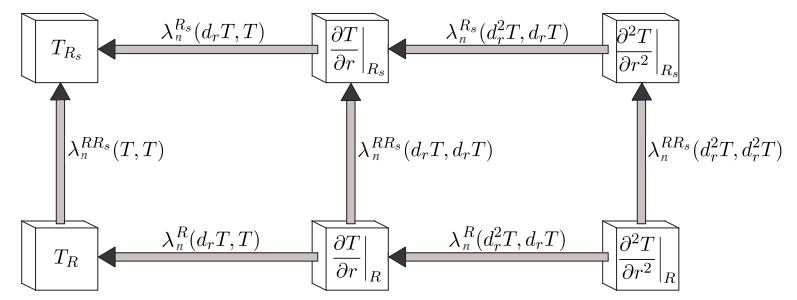

Fig. 1 The spherical Meissl scheme (adapted from Rummel and Van Gelderen 1995)

$$
\begin{aligned}
& \lambda_{n}^{R}\left(d_{r} T, T\right) \quad=\frac{-R}{n+1} \quad \lambda_{n}^{R R_{\mathrm{s}}}(T, T) \quad=\left(\frac{R}{R_{\mathrm{s}}}\right)^{n+1} \\
& \lambda_{n}^{R_{\mathrm{s}}}\left(d_{r} T, T\right)=\frac{-R_{\mathrm{s}}}{n+1} \quad \lambda_{n}^{R R_{\mathrm{s}}}\left(d_{r} T, d_{r} T\right)=\left(\frac{R}{R_{\mathrm{s}}}\right)^{n+2} \\
& \lambda_{n}^{R}\left(d_{r}^{2} T, d_{r} T\right)=\frac{-R}{n+2} \quad \lambda_{n}^{R R_{\mathrm{S}}}\left(d_{r}^{2} T, d_{r}^{2} T\right)=\left(\frac{R}{R_{\mathrm{s}}}\right)^{n+3} \\
& \lambda_{n}^{R_{\mathrm{s}}}\left(d_{r}^{2} T, d_{r} T\right)=\frac{-R_{\mathrm{s}}}{n+2}
\end{aligned}
$$

Table 1 The seven eigenvalues of the spherical Meissl scheme (Fig. 1)

The arrows in Fig. 1 point in the direction of decreased power in the high frequencies, showing that the lower frequencies become more dominant for higher altitudes and for lower-order derivatives (a power-shift filtering process). For instance, geoid heights have more power in the low frequencies than gravity anomalies.

\section{The Meissl scheme for the geodetic ellipsoid}

The original [spherical] Meissl scheme can be generalised to not only transform between spherical harmonic expansions defined with respect to spheres, but more generally between spherical harmonic expansions defined with respect to an oblate ellipsoid of revolution (i.e., geodetic ellipsoids).

Furthermore, the first- and second-order radial derivatives in the spherical Meissl scheme can be replaced by firstand second-order derivatives with respect to the ellipsoidal normal $(h)$, which are often of more interest than the radial derivatives. Such a generalisation results in a Meissl scheme for the ellipsoid, albeit in terms of spherical harmonics (see Fig. 2).

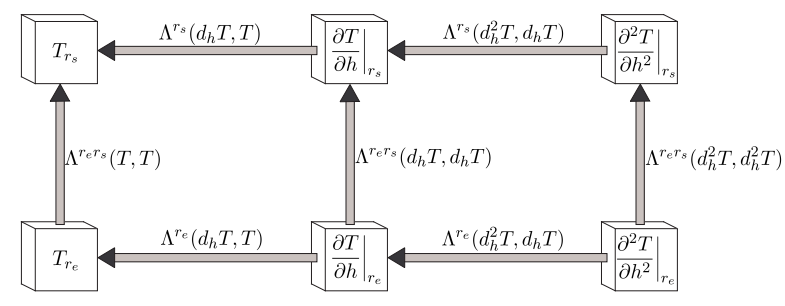

Fig. 2 The Meissl scheme for the geodetic ellipsoid

In the case that the quantities are not given on a sphere, but on an ellipsoid, surface spherical harmonic expansions can still be computed (e.g., Jekeli 1988). However, one-toone relations between the surface SHCs no longer exist. Instead, SHCs of one quantity can be expressed as a weighted summation over SHCs of another quantity. The details of these weighted summations are provided in Section 4.

Due to the rotational symmetry of the geodetic ellipsoid, computation of a SHC of degree $n$ and order $m$ only requires SHCs of the original quantity of the same order $m$. The general transformation formula is (cf. Eq. 4)

$$
{\overline{d_{h}^{j} T}}_{n m}^{\mathcal{R}_{2}}=\sum_{k=-\infty}^{\infty} \lambda_{n m k}^{\mathcal{R}_{1} \mathcal{R}_{2}}\left(d_{h}^{i} T, d_{h}^{j} T\right){\overline{d_{h}^{i} T}}_{n-2 k, m}^{\mathcal{R}_{1}},
$$

$$
0 \leq i, j \leq 2
$$

Compared to Eq. (4), the radial derivatives are replaced by normal-to-the-ellipsoid derivatives (symbolised by the subscript $h$ ), and the radii $R_{1}$ and $R_{2}$ are replaced by $\mathcal{R}_{1}$ and $\mathcal{R}_{2}$, which symbolise the ellipsoid of the original quantity and the ellipsoid of the desired quantity, respectively. Furthermore, a summation over SHCs of odd or even degrees $n$ is included.

Equation (9) can also be written in matrix form $d_{h}^{j} \mathcal{T}^{\mathcal{R}_{2}}=\Lambda^{\mathcal{R}_{1} \mathcal{R}_{2}}\left(d_{h}^{i} T, d_{h}^{j} T\right) d_{h}^{i} \mathcal{T}^{\mathcal{R}_{1}}, \quad 0 \leq i, j \leq 2$ where $d_{h}^{i} \mathcal{T}^{\mathcal{R}_{1}}$ is a vector containing all SHCs of the original quantity, $d_{h}^{j} \mathcal{T}^{\mathcal{R}_{2}}$ is a vector containing all SHCs of the desired quantity, and $\Lambda^{\mathcal{R}_{1} \mathcal{R}_{2}}\left(d_{h}^{i} T, d_{h}^{j} T\right)$ is the transformation matrix, containing the transformation factors $\lambda_{n m i}^{\mathcal{R}_{1} \mathcal{R}_{2}}\left(d_{h}^{i} T, d_{h}^{j} T\right)$. The transformation matrix in Eq. (10) is block-diagonal if the SHCs in the two vectors $d_{h}^{i} \mathcal{T}^{\mathcal{R}_{1}}$ and $d_{h}^{j} \mathcal{T}^{\mathcal{R}_{2}}$ are ordered first by order $m$ and subsequently by even or odd degree $n$.

The one-to-one relations of the spherical Meissl scheme are thus replaced by matrix transformations in the Meissl 
scheme for the geodetic ellipsoid (Fig. 2). Like the spherical Meissl scheme, our Meissl scheme shows the case of an ellipsoid at 'ground level', symbolised by the ellipsoidal radius $r_{\mathrm{e}}$, and the case of an ellipsoid at 'satellite height', symbolised by the ellipsoidal radius $r_{\mathrm{s}}$ (cf. Rummel and Van Gelderen 1995). The two ellipsoids with radii $r_{\mathrm{e}}$ and $r_{\mathrm{s}}$ do not necessarily need to have the same eccentricity $e$ (or flattening $f$ ), although from a numerical point of view, the eccentricity of both should be small (see Section 5). It is, however, assumed that both ellipsoids have the same origin and rotation axis.

Finally, in the limiting case that both ellipsoids have an eccentricity of zero, the Meissl scheme for the ellipsoid degenerates to the spherical Meissl scheme. The transformation matrices will then all become diagonal matrices, with the eigenvalues from Table 1 along the diagonal.

\section{Computation of the transformation matrices $\Lambda$}

The computation of the transformation matrices in the Meissl scheme for the ellipsoid (Fig. 2) is not straightforward. However, derivation of only three transformation matrices is sufficient to compute all others via simple matrix multiplications. This follows from the fact that the transformations can be applied successively, which results in a relation among the transformation matrices

$$
\begin{array}{r}
\Lambda^{\mathcal{R}_{1} \mathcal{R}_{2}}\left(d_{h}^{j} T, d_{h}^{i} T\right)=\Lambda^{\mathcal{R}_{1} \mathcal{R}_{3}}\left(d_{h}^{k} T, d_{h}^{i} T\right) \\
\quad \times \Lambda^{\mathcal{R}_{3} \mathcal{R}_{2}}\left(d_{h}^{j} T, d_{h}^{k} T\right), \quad 0 \leq i, j, k \leq 2
\end{array}
$$

It is thus possible to compute a transformation matrix for transformations between two ellipsoids with different eccentricities by consecutive transformations to and from a sphere.

Because a reverse transformation can be found by inversion of the transformation matrix, it also holds that

$\Lambda^{\mathcal{R}_{1} \mathcal{R}_{2}}\left(d_{h}^{j} T, d_{h}^{i} T\right)=\left[\Lambda^{\mathcal{R}_{2} \mathcal{R}_{1}}\left(d_{h}^{i} T, d_{h}^{j} T\right)\right]^{-1}$

but it should be noted that the inversion can only be applied if the transformation matrix is square and numerically wellconditioned. The matrix will always be square if the maximum degree and order of the desired set of harmonics is chosen equal to the maximum degree and order of the original set. The question of whether the matrix is well- or illconditioned is answered in Section 5.

Instead of deriving any of the seven relations in the Meiss scheme (Fig. 2), three auxiliary transformations can be derived, namely the relations between solid SHCs of $T$ and surface SHCs of $T$ and its first- and second-order derivatives with respect to $h$. In other words, the matrices $\Lambda^{R r_{\mathrm{e}}}(T, T)$, $\Lambda^{R r_{\mathrm{e}}}\left(T, d_{h} T\right)$ and $\Lambda^{R r_{\mathrm{e}}}\left(T, d_{h}^{2} T\right)$ are derived, and all matrices in the Meissl scheme for the geodetic ellipsoid can be derived from these matrices using Eqs. (11) and (12).
As an example, the transformation matrix $\Lambda^{r_{\mathrm{e}}}\left(d_{h}^{2} T, d_{h} T\right)$ that computes first-order derivatives from second-order derivatives on the ellipsoid follows from

$\Lambda^{r_{\mathrm{e}}}\left(d_{h}^{2} T, d_{h} T\right)=\left[\Lambda^{R r_{\mathrm{e}}}\left(T, d_{h}^{2} T\right)\right]^{-1} \Lambda^{R r_{\mathrm{e}}}\left(T, d_{h} T\right)$ The three matrices $\Lambda^{R r_{\mathrm{e}}}(T, T), \Lambda^{R r_{\mathrm{e}}}\left(T, d_{h} T\right)$ and $\Lambda^{R r_{\mathrm{e}}}\left(T, d_{h}^{2} T\right)$ form a connection between the spherical Meissl scheme and the Meissl scheme for the ellipsoid (Fig. 3). The connections at 'ground level' also hold at 'satellite height'.

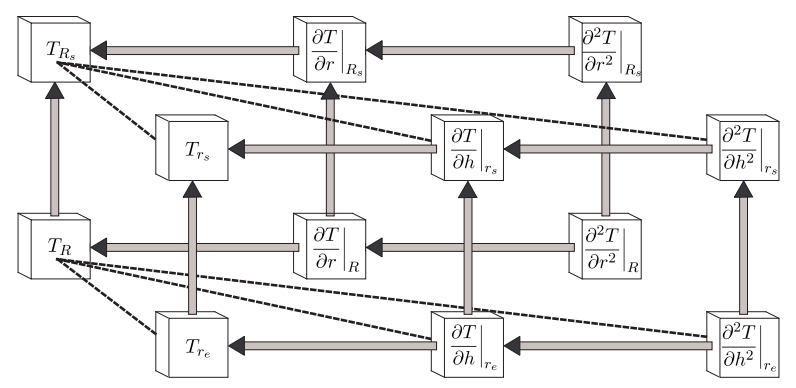

Fig. 3 Connections among the spherical and ellipsoidal Meissl schemes (black dashed lines), given by Eqs. (20), (43) and (44)

The task of finding the transformation matrix $\Lambda^{R r_{\mathrm{e}}}(T, T)$ comes down to finding a general transformation between solid and surface SHCs. Assuming that $T$ is harmonic everywhere outside the surface of the ellipsoid, the solid and surface spherical harmonic expansions can be equated

$$
\begin{aligned}
T\left(r_{\mathrm{e}}, \theta, \lambda\right) & =\sum_{n=0}^{\infty} \sum_{m=-n}^{n}\left(\frac{R}{r_{\mathrm{e}}(\theta)}\right)^{n+1} \bar{T}_{n m}^{R} \bar{Y}_{n m}(\theta, \lambda) \\
& =\sum_{n=0}^{\infty} \sum_{m=-n}^{n} \bar{T}_{n m}^{r_{\mathrm{e}}} \bar{Y}_{n m}(\theta, \lambda)
\end{aligned}
$$

The presence of the ellipsoidal radius $r_{\mathrm{e}}$ on the left-hand side of Eq. (14) destroys the orthogonality of the spherical harmonic basis functions, and a one-to-one relation between the coefficients of each pair of degree $n$ and order $m$ therefore does not exist. A relation between both coefficients independent of longitude $\lambda$ and geocentric co-latitude $\theta$ can nevertheless be found when the dependence on $\theta$ is moved inside the spherical harmonic functions $\bar{Y}_{n m}$.

This is achieved when the term $\left(R / r_{\mathrm{e}}\right)^{n+1}$ is expanded into a binomial series

$T\left(r_{\mathrm{e}}, \theta, \lambda\right)=\sum_{n=0}^{\infty} \sum_{m=-n}^{n} \sum_{j=0}^{\infty} \alpha_{n j} \sin ^{2 j} \theta \bar{T}_{n m}^{R} \bar{Y}_{n m}$

where

$\alpha_{n j}=\left(\frac{c}{\sqrt{1-e^{2}}}\right)^{n+1}(-1)^{j}\left(\begin{array}{c}\frac{n+1}{2} \\ j\end{array}\right) e^{2 j}$

In Eq. (16), $c=a / R$, i.e., the ratio between the semi-major axis $a$ of the ellipsoid and the reference sphere radius $R$, and $e^{2}$ is the square of the first numerical eccentricity of the oblate ellipsoid. Since for any ellipsoid $0 \leq e^{2} \sin ^{2} \theta<1$, the binomial series will always be alternating and convergent. 
Equation (15) contains the product of an even power of the sine of the geocentric co-latitude $\theta$ and the spherical harmonic functions, which can be equated to a weighted summation over spherical harmonic functions of the same order $m$ (Claessens 2005)

$\sin ^{2 j} \theta \bar{Y}_{n m}=\sum_{i=-j}^{j} \bar{K}_{n m}^{2 i, 2 j} \bar{Y}_{n+2 i, m}$

Spherical harmonic functions of negative degree should be set equal to zero in the evaluation of Eq. (17) and other equations hereafter. The weights $\bar{K}_{n m}^{2 i, 2 j}$ depend only on the spherical harmonic degree $n$, order $m$ and the summation indices $i$ and $j$. These weights can be computed efficiently through an iterative scheme (Claessens 2005)

$\bar{K}_{n m}^{2 i, 2 j}=\sum_{k=-1}^{1} \bar{K}_{n m}^{2 k, 2} \bar{K}_{n+2 k, m}^{2(i-k), 2(j-1)}$

based on the initial values

$$
\begin{aligned}
& \bar{K}_{n m}^{-2,2}=-\sqrt{\frac{\left[(n-1)^{2}-m^{2}\right]\left(n^{2}-m^{2}\right)}{(2 n-3)(2 n-1)^{2}(2 n+1)}} \\
& \bar{K}_{n m}^{0,2}=\frac{2\left(n^{2}+m^{2}+n-1\right)}{(2 n-1)(2 n+3)} \\
& \bar{K}_{n m}^{2,2}=-\sqrt{\frac{\left[(n+1)^{2}-m^{2}\right]\left[(n+2)^{2}-m^{2}\right]}{(2 n+1)(2 n+3)^{2}(2 n+5)}}
\end{aligned}
$$

Inserting Eq. (17) into Eq. (15) and reorganising the order of summations allows for the one-to-one comparison of the SHCs, ultimately leading to the final transformation formula, which includes an infinite summation

$\bar{T}_{n m}^{r_{\mathrm{e}}}=\sum_{i=-\infty}^{\infty} \lambda_{n m i}^{R r_{\mathrm{e}}}(T, T) \bar{T}_{n-2 i, m}^{R}$

SHCs of negative degree should be set equal to zero in the evaluation of Eq. (20) and other equations hereafter. The weights in Eq. (20) also follow from an infinite summation

$\lambda_{n m i}^{R r_{\mathrm{e}}}(T, T)=\sum_{j=|i|}^{\infty} \alpha_{n-2 i, j} \bar{K}_{n-2 i, m}^{2 i, 2 j}$

Both infinite summations can, in practice, be confined to a finite range because the series converge rapidly for ellipsoids with a small eccentricity, like the geodetic ellipsoid ( $e^{2}=0.00669438002290$; Moritz 1980b). For SHCs up to degree and order 360 , it is sufficient to run the summation in Eq. (20) from $i=-20$ to $i=20$, and the summation in Eq. (21) up to $j=20$, in which case the relative truncation error will be $<10^{-8}$. This requires less than 1 minute of CPU time on a Sun UltraSPARC III Cu $1.2 \mathrm{GHz}$ processor with $8 \mathrm{~GB}$ of RAM.

However, for higher degrees and orders, the range of summation needs to be extended. Roughly, the maximum values of $|i|$ and $j$ need to be increased by one for every increase in spherical harmonic degree $n$ of $\sim 50$ to obtain a similar relative truncation error. Thus, for SHCs up to degree and order 2160, the summation in Eq. (20) should be performed from $i=-56$ to $i=56$, and the summation in
Eq. (21) up to $j=56$ to obtain a relative truncation error $<10^{-8}$.

Equation (20) is a special case of Eq. (9) and can also be written in matrix form. The weights $\lambda_{n m i}^{R r_{\mathrm{e}}}(T, T)$ then form the elements of the matrix $\Lambda^{R r_{\mathrm{e}}}(T, T)$. Derivation of the matrices $\Lambda^{R r_{\mathrm{e}}}\left(T, d_{h} T\right)$ and $\Lambda^{R r_{\mathrm{e}}}\left(T, d_{h}^{2} T\right)$, which define the transformation between solid SHCs and surface SHCs of the first- and second-order normal derivative on an ellipsoid, can be achieved along similar lines as the derivation of $\Lambda^{R r_{\mathrm{e}}}(T, T)$. However, the complexity of the transformation formulas increases significantly for the higher derivatives, as follows.

The first- and second-order derivatives of $T$ with respect to $h$ can be expressed in terms of the radial and latitudinal derivatives. For points on the surface of the ellipsoid, it degenerates to (Jekeli 1981)

$$
\begin{aligned}
& \frac{\partial T}{\partial h}=\cos \phi \frac{\partial T}{\partial r}-\sin \phi \frac{\partial T}{r_{\mathrm{e}} \partial \theta} \\
& \text { and (Claessens 2006) } \\
& \frac{\partial^{2} T}{\partial h^{2}}=\cos ^{2} \phi \frac{\partial^{2} T}{\partial r^{2}}+\sin ^{2} \phi\left(\frac{\partial T}{r_{\mathrm{e}} \partial r}+\frac{\partial^{2} T}{r_{\mathrm{e}}^{2} \partial \theta^{2}}\right) \\
& +\sin 2 \phi\left(\frac{\partial T}{r_{\mathrm{e}}^{2} \partial \theta}-\frac{\partial^{2} T}{r_{\mathrm{e}} \partial r \partial \theta}\right)
\end{aligned}
$$

where $\phi$ is the difference between the geocentric co-latitude $\theta$ and the geodetic co-latitude $\vartheta(\phi=\theta-\vartheta)$. The sine and cosine of $\phi$ can be written as a function of the geocentric co-latitude as (Claessens 2006, p. 20)

$$
\begin{aligned}
& \sin \phi=\frac{e^{2} \sin \theta \cos \theta}{\sqrt{1-e^{2}\left(2-e^{2}\right) \sin ^{2} \theta}} \\
& \cos \phi=\frac{1-e^{2} \sin ^{2} \theta}{\sqrt{1-e^{2}\left(2-e^{2}\right) \sin ^{2} \theta}}
\end{aligned}
$$

The solid spherical harmonic expansion of $T$ (Eq. 1) is inserted into Eqs. (22) and (23) to yield (Claessens 2006)

$$
\begin{array}{r}
\frac{\partial T}{\partial h}=-\frac{1}{R} \sum_{n=0}^{\infty}\left(\frac{R}{r_{\mathrm{e}}}\right)^{n+2} \sum_{m=-n}^{n} \bar{T}_{n m}^{R}[(n+1) \cos \phi \\
\left.+\sin \phi \frac{\partial}{\partial \theta}\right] \bar{Y}_{n m}
\end{array}
$$

$$
\begin{aligned}
\frac{\partial^{2} T}{\partial h^{2}}=\frac{1}{R^{2}} \sum_{n=0}^{\infty}\left(\frac{R}{r_{\mathrm{e}}}\right)^{n+3} \sum_{m=-n}^{n} \bar{T}_{n m}^{R}\{(n+1) \\
\times\left[n+2-(n+3) \sin ^{2} \phi\right]+(n+2) \sin 2 \phi \frac{\partial}{\partial \theta} \\
\left.+\sin ^{2} \phi \frac{\partial^{2}}{\partial \theta^{2}}\right\} \bar{Y}_{n m}
\end{aligned}
$$

The co-latitudinal derivatives of the spherical harmonic functions in Eqs. (26) and (27) can be removed using two more relations among spherical harmonics, as follows.

The first latitudinal derivative of the spherical harmonic functions can be rewritten as (e.g., Moritz 1980a; Sjöberg 2003; Claessens 2005)

$\sin \theta \cos \theta \frac{\partial \bar{Y}_{n m}}{\partial \theta}=\bar{N}_{n, m,-2} \bar{Y}_{n-2, m}+\bar{N}_{n, m, 0} \bar{Y}_{n m}$ 
$+\bar{N}_{n, m, 2} \bar{Y}_{n+2, m}$

where

$\bar{N}_{n, m,-2}=(n+1) \bar{K}_{n m}^{-2,2}$

$\bar{N}_{n, m, 0}=\frac{3}{2} \bar{K}_{n m}^{0,2}-1$

$\bar{N}_{n, m, 2}=-n \bar{K}_{n m}^{2,2}$

The second co-latitudinal derivative of the spherical harmonic functions follows from the characteristic differential equation (e.g., Hobson 1931)

$\sin ^{2} \theta \frac{\partial^{2} \bar{Y}_{n m}}{\partial \theta^{2}}=-\sin \theta \cos \theta \frac{\partial \bar{Y}_{n m}}{\partial \theta}-\left[n(n+1) \sin ^{2} \theta\right.$

which after insertion of Eqs. (18) and (28) reads

$$
\begin{array}{r}
\sin ^{2} \theta \frac{\partial^{2} \bar{Y}_{n m}}{\partial \theta^{2}}=\bar{R}_{n, m,-2} \bar{Y}_{n-2, m}+\bar{R}_{n, m, 0} \bar{Y}_{n m} \\
+\bar{R}_{n, m, 2} \bar{Y}_{n+2, m}
\end{array}
$$

where

$\begin{aligned} \bar{R}_{n, m, 0} & =-\left(n^{2}+n+\frac{3}{2}\right) \bar{K}_{n m}^{0,2}+m^{2}+1\end{aligned}$

$\bar{R}_{n, m, 2}=-n^{2} \bar{K}_{n m}^{2,2}$

Inserting Eqs. (28) and (31) into Eqs. (26) and (27) yields the first- and second-order normal derivatives of $T$ with respect to $h$ as a function of solid SHCs and spherical harmonic basis functions

$$
\begin{aligned}
\frac{\partial T}{\partial h}= & -\frac{1}{R} \sum_{n=0}^{\infty}\left(\frac{R}{r_{\mathrm{e}}}\right)^{n+2} \sum_{m=-n}^{n} \bar{T}_{n m}^{R}[(n+1) \cos \phi \\
& \left.\times \bar{Y}_{n m}+\frac{\sin \phi}{\sin \theta \cos \theta} \sum_{p=-1}^{1} \bar{N}_{n m, 2 p} \bar{Y}_{n+2 p, m}\right]
\end{aligned}
$$

and

$$
\begin{gathered}
\frac{\partial^{2} T}{\partial h^{2}}=\frac{1}{R^{2}} \sum_{n=0}^{\infty}\left(\frac{R}{r_{\mathrm{e}}}\right)^{n+3} \sum_{m=-n}^{n} \bar{T}_{n m}^{R}\{(n+1)[n+2 \\
\left.-(n+3) \sin ^{2} \phi\right] \bar{Y}_{n m}+(n+2) \frac{\sin 2 \phi}{\sin \theta \cos \theta} \sum_{p=-1}^{1} \\
\left.\bar{N}_{n m, 2 p} \bar{Y}_{n+2 p, m}+\frac{\sin ^{2} \phi}{\sin ^{2} \theta} \sum_{p=-1}^{1} \bar{R}_{n m, 2 p} \bar{Y}_{n+2 p, m}\right\}
\end{gathered}
$$

All terms that include the [small] angle $\phi$ can be expanded into binomial series. The sum of two series can be combined via component-wise addition and the product of two binomial series can be expressed as one binomial series using a Cauchy multiplication (e.g., Protter and Morrey 1964).

From Eqs. (24) and (25), it follows that

$\cos \phi=\sum_{k=0}^{\infty}(-1)^{k} e^{2 k}\left[\sum_{l=k-1}^{k}\left(2-e^{2}\right)^{l}\left(\begin{array}{c}-\frac{1}{2} \\ l\end{array}\right)\right] \sin ^{2 k} \theta$

$\frac{\sin \phi}{\sin \theta \cos \theta}=e^{2} \sum_{k=0}^{\infty}(-1)^{k} e^{2 k}\left(2-e^{2}\right)^{k}\left(\begin{array}{c}-\frac{1}{2} \\ k\end{array}\right) \sin ^{2 k} \theta$

$\sin ^{2} \phi=e^{4} \sum_{k=0}^{\infty}(-1)^{k+1}\left[\sum_{l=k-2}^{k-1} e^{2 l}\left(2-e^{2}\right)^{l}\left(\begin{array}{c}-1 \\ l\end{array}\right)\right]$

$$
\times \sin ^{2 k} \theta
$$

$$
\begin{array}{r}
\frac{\sin 2 \phi}{\sin \theta \cos \theta}=2 e^{2} \sum_{k=0}^{\infty}(-1)^{k} e^{2 k}\left[\sum_{l=k-1}^{k}\left(2-e^{2}\right)^{l}\left(\begin{array}{c}
-1 \\
l
\end{array}\right)\right] \\
\times \sin ^{2 k} \theta
\end{array}
$$

$\frac{\sin ^{2} \phi}{\sin ^{2} \theta}=e^{4} \sum_{k=0}^{\infty}(-1)^{k}\left[\sum_{l=k-1}^{k} e^{2 l}\left(2-e^{2}\right)^{l}\left(\begin{array}{c}-1 \\ l\end{array}\right)\right] \sin ^{2 k} \theta$

The five series in Eq. (35) can all be combined with the binomial series of the terms $\left(R / r_{\mathrm{e}}\right)^{n+2}$ or $\left(R / r_{\mathrm{e}}\right)^{n+3}$ using Cauchy multiplication, and the combined series can then be inserted into Eqs. (33) and (34). Subsequently, Eq. (17) can be used to shift all dependence on $\theta$ into $\bar{Y}_{n m}$, introducing an additional summation over index $i$.

After rearrangement of the summations over $i$ and $p$, Eqs. (33) and (34) become

$\frac{\partial T}{\partial h}=-\frac{1}{R} \sum_{n=0}^{\infty} \sum_{m=-n}^{n} \bar{T}_{n m}^{R} \sum_{j=0}^{\infty} \sum_{i=-j-1}^{j+1}\left[(n+1) \beta_{n m i j}\right.$

$$
\left.+\gamma_{n m i j}\right] \bar{Y}_{n+2 i, m}
$$

and

$\frac{\partial^{2} T}{\partial h^{2}}=\frac{1}{R^{2}} \sum_{n=0}^{\infty} \sum_{m=-n}^{n} \bar{T}_{n m}^{R} \sum_{j=0}^{\infty} \sum_{i=-j-1}^{j+1}\left[(n+1)(n+2) \delta_{0 i}\right.$ $\left.-(n+1)(n+3) \kappa_{n m i j}+(n+2) \zeta_{n m i j}+\eta_{n m i j}\right] \bar{Y}_{n+2 i, m}$

where $\delta_{0 i}$ is the Kronecker delta and

$$
\begin{gathered}
\beta_{n m i j}=\bar{K}_{n m}^{2 i, 2 j} \sum_{k=0}^{j} \alpha_{n+1, j-k}(-1)^{k} e^{2 k} \\
\times \sum_{l=k-1}^{k}\left(2-e^{2}\right)^{l}\left(\begin{array}{c}
-\frac{1}{2} \\
l
\end{array}\right) \\
\gamma_{n m i j}=e^{2}\left[\sum_{p=i-1}^{i+1} \bar{K}_{n+2(i-p), m}^{2 p, 2 j} \bar{N}_{n m, 2(i-p)}\right] \\
\times \sum_{k=0}^{j} \alpha_{n+1, j-k}(-1)^{k} e^{2 k}\left(2-e^{2}\right)^{k}\left(\begin{array}{c}
-\frac{1}{2} \\
k
\end{array}\right)
\end{gathered}
$$

$\kappa_{n m i j}=e^{4} \bar{K}_{n m}^{2 i, 2 j} \sum_{k=0}^{j} \alpha_{n+2, j-k}(-1)^{k+1}$

$$
\times \sum_{l=k-2}^{k-1} e^{2 l}\left(2-e^{2}\right)^{l}\left(\begin{array}{c}
-1 \\
l
\end{array}\right)
$$

$\zeta_{n m i j}=2 e^{2}\left[\sum_{p=i-1}^{i+1} \bar{K}_{n+2(i-p), m}^{2 p, 2 j} \bar{N}_{n m, 2(i-p)}\right]$

$\times \sum_{k=0}^{j} \alpha_{n+2, j-k}(-1)^{k} e^{2 k} \sum_{l=k-1}^{k}\left(2-e^{2}\right)^{l}\left(\begin{array}{c}-1 \\ l\end{array}\right)$ 
$\times \sum_{k=0}^{j} \alpha_{n+2, j-k}(-1)^{k} \sum_{l=k-1}^{k} e^{2 l}\left(2-e^{2}\right)^{l}\left(\begin{array}{c}-1 \\ l\end{array}\right)$

In the numerical evaluation of Eq. (38), $\bar{K}_{n+2(i-p), m}^{2 p, 2 j}$ should be set to zero when $|p|>j$.

The summation order over $n$ and $i$ in Eqs. (36) and (37) can be rearranged to yield expressions where the spherical harmonic functions depend solely on degree $n$ and order $m$. Subsequently, the summations over indices $i$ and $j$ can be reorganised so that the summation over $i$ is brought outside the summation over $j$. Then, a comparison between the solid SHCs and surface SHCs of the first- and second-order normal derivative can be achieved.

The final transformation formulas read

${\overline{d_{h} T}}_{n m}^{r_{\mathrm{e}}}=\sum_{i=-\infty}^{\infty} \lambda_{n m i}^{R r_{\mathrm{e}}}\left(d_{h} T, T\right) \bar{T}_{n-2 i, m}^{R}$

and

${\overline{d_{h}^{2} T}}_{n m}^{r_{\mathrm{e}}}=\sum_{i=-\infty}^{\infty} \lambda_{n m i}^{R r_{\mathrm{e}}}\left(d_{h}^{2} T, T\right) \bar{T}_{n-2 i, m}^{R}$

where the weights $\lambda_{n m i}^{R r_{\mathrm{e}}}\left(d_{h} T, T\right)$ and $\lambda_{n m i}^{R r_{\mathrm{e}}}\left(d_{h}^{2} T, T\right)$ are given by

$$
\begin{array}{r}
\lambda_{n m i}^{R r_{\mathrm{e}}}\left(d_{h} T, T\right)=-\frac{1}{R} \sum_{j=|i|-1}^{\infty}\left[(n-2 i+1) \beta_{n-2 i, m i j}\right. \\
\left.+\gamma_{n-2 i, m i j}\right]
\end{array}
$$

and

$$
\begin{array}{r}
\lambda_{n m i}^{R r_{\mathrm{e}}}\left(d_{h}^{2} T, T\right)=\frac{1}{R^{2}} \sum_{j=|i|-1}^{\infty}\left[(n+1)(n+2) \delta_{0 i}\right. \\
-(n-2 i+1)(n-2 i+3) \kappa_{n-2 i, m i j} \\
\left.+(n-2 i+2) \zeta_{n-2 i, m i j}+\eta_{n-2 i, m i j}\right]
\end{array}
$$

Thus, the weights given by Eqs. (21), (45) and (46) only depend upon the degree $n$, order $m$, index $i$, reference sphere radius $R$ and the semi-major axis $a$ and eccentricity $e$ of the ellipsoid. These weights populate the transformation matrices $\Lambda^{R r_{\mathrm{e}}}(T, T), \Lambda^{R r_{\mathrm{e}}}\left(T, d_{h} T\right)$ and $\Lambda^{R r_{\mathrm{e}}}\left(T, d_{h}^{2} T\right)$ that connect the spherical Meissl scheme and the Meissl scheme for the ellipsoid (the dashed lines in Fig. 3). Using these transformation matrices in combination with Eqs. (8) and (9), any transformation within the ellipsoidal Meissl scheme (Fig. 2) can be performed.

\section{Forward and reverse transformations}

Equations (20), (43) and (44) can be used to compute surface SHCs defined with respect to an ellipsoid from a set of solid SHCs. These transformations can be performed with high accuracy to at least degree and order 2160, which is consistent with the expected degree and order of the EGM07 global geopotential model (Pavlis et al., 2007).

However, the reverse transformation requires the inversion of the transformation matrix. This inversion is only possible if the matrix is square and numerically well-conditioned.
The matrix will always be square if the maximum degree and order of the two sets of coefficients are chosen to be equal.

The question whether the matrix will be numerically wellconditioned can only be answered affirmatively up to a certain degree and order, as follows. The small eccentricity $e$ of the geodetic ellipsoid will result in a transformation matrix that is strictly diagonally dominant (i.e., the absolute value of each diagonal element is larger then the sum of all absolute values of other elements in that row) for a large range of degrees and orders. In this case, the matrix is always wellconditioned.

An additional advantage of the diagonal dominance of the transformation matrix is that inversion can be achieved efficiently by an iterative approach, such as Jacobi or GaussSeidel iteration (e.g., Strang 1986). These iteration schemes will always converge if the criterion of diagonal dominance is met, and the convergence becomes more rapid as the diagonal dominance gets stronger. However, once the diagonal dominance is lost, the transformation matrix becomes ill-conditioned and the inversion becomes inaccurate.

Figure 4 shows a measure of the diagonal dominance of the transformation matrix $\Lambda^{R r_{\mathrm{e}}}(T, T)$ for an ellipsoid with the eccentricity of the Earth and for coefficients up to $n=$ 720 . Beyond degree $n=520$, some rows in the transformation matrix are no longer diagonally dominant, especially for low $m$. The transformation matrices for the higher-order derivatives, $\Lambda^{R r_{\mathrm{e}}}\left(d_{h} T, T\right)$ and $\Lambda^{R r_{\mathrm{e}}}\left(d_{h}^{2} T, T\right)$, show a very similar pattern.

Since all transformations in the Meissl scheme for the ellipsoid (Fig. 2) are derived from a subsequent application of a reverse and a forward transformation, all will suffer from the loss of diagonal dominance beyond degree 520 . However, in the case of transformations among $T$ and its derivatives at the surface of the same ellipsoid, visualised by the horizontal arrows in the Meissl scheme for the ellipsoid (Fig. 2), these numerical problems can be avoided.

As an example, consider the relation between the surface SHCs of $T$ and the surface SHCs of its normal derivative $\partial T / \partial h$. The normal derivative of $T$ can be written in terms of the surface SHCs $\bar{T}_{n m}^{r_{\mathrm{e}}}$ as

$$
\begin{array}{r}
\frac{\partial T}{\partial h}=-\frac{1}{r_{\mathrm{e}}} \sum_{n=0}^{\infty} \sum_{m=-n}^{n} \bar{T}_{n m}^{r_{\mathrm{e}}}\left((n+1) \cos \phi \bar{Y}_{n m}\right. \\
\left.+\sin \phi \frac{\partial \bar{Y}_{n m}}{\partial \theta}\right)
\end{array}
$$

The only difference between Eqs. (33) and (47), besides the change from solid to surface SHCs, is that the term $\left(R / r_{\mathrm{e}}\right)$ is not raised to the power $(n+2)$. A relation between the surface SHCs of $T$ and those of its normal derivative can therefore be derived along exactly the same lines as laid out in Section 4. 


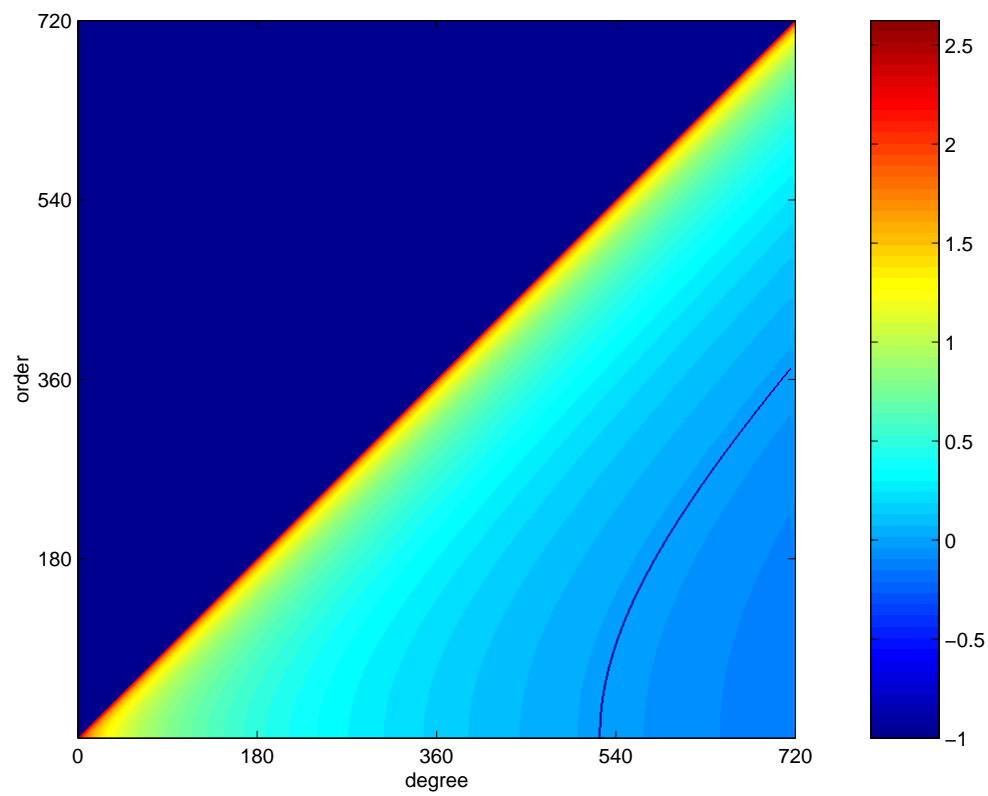

Fig. 4 Logarithm of the ratio between $\left|\lambda_{n m 0}\right|$ and $\sum_{i=-50, i \neq 0}^{50}\left|\lambda_{n m i}\right|$ for all degrees $n$ and orders $m$ up to 720 , where the line starting at degree $n=520$ indicates for which pairs of $n$ and $m$ the ratio is closest to unity

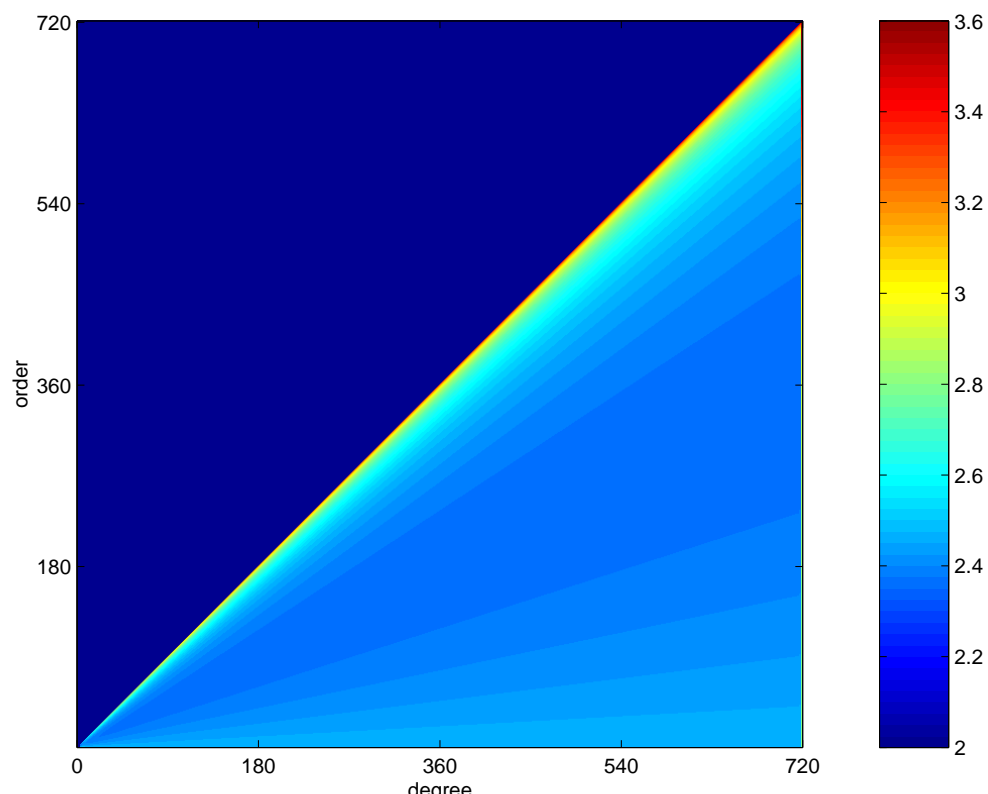

Fig. 5 Logarithm of the ratio between $\left|\lambda_{n m 0}\left(d_{h} T, T\right)\right|$ and $\sum_{i=-50, i \neq 0}^{50}\left|\lambda_{n m i}\left(d_{h} T, T\right)\right|$ for a transformation between surface SHCs of a function and its normal derivative on the ellipsoid $(0 \leq n \leq 720)$ 
In this case, the binomial coefficients are no longer dependent on the degree $n$, which means that the weights of $\lambda_{n m i}$ of $i \neq 0$ (the off-diagonal weights) do not significantly increase for higher degrees $n$. The matrix containing the weights is therefore strongly diagonally dominant, even when SHCs of very high degree and order are taken into account (Fig. 5). This not only makes the forward transformation more efficient, but also ensures that iterative procedures for the reverse transformation will always converge.

The rate of convergence of these iterative methods will be high, due to the strong diagonal dominance displayed in Fig. 5, with every element on the diagonal being at least two orders of magnitude larger than the sum of all other elements in the same row.

The spectral relation between the surface SHCs of $T$ and its second normal derivative can be obtained along the same principles, and all horizontal transformations in the Meissl scheme for the ellipsoid (Fig. 2) can thus be performed very efficiently in both directions. Transformations from the sphere to the ellipsoid are less efficient, due to the increase in the off-diagonal terms for higher degrees $n$, but only contain a forward transformation and do therefore not contain the problems encountered in the reverse transformations.

Only a transformation from an ellipsoidal surface to another surface (either a sphere or another ellipsoid) requires the inversion of a non-diagonally dominant matrix, which can only be performed efficiently up to degree and order 520 for an ellipsoid with the eccentricity of the Earth.

\section{Conclusion}

The Meissl scheme for the geodetic ellipsoid provides the spectral relations among the disturbing potential $T$ and its first- and second-order normal derivatives with respect to $h$ on any ellipsoid, also allowing transformations from one ellipsoid to another. The transformations are expressed as a multiplication of a block-diagonal matrix with a vector containing surface SHCs of one quantity, yielding a vector containing surface SHCs of a second quantity.

Numerical instabilities may occur in the transformation from the ellipsoid to another surface for spherical harmonic expansions beyond degree 520, because this requires inversion of an ill-posed, non-diagonally dominant matrix. All other transformations in the scheme are highly accurate and efficient up to at least degree and order 2160, which is the same as the forthcoming EGM07 global geopotential model.

Ultimately, a fully ellipsoidal Meissl scheme using ellipsoidal harmonics may overcome the numerical difficulties in the very high degrees faced in the present formulation using surface spherical harmonics on the geodetic ellipsoid, but this still requires further development and implementation.
However, this must be countered with the numerical instabilities in ellipsoidal harmonics for high degrees (cf. Sona 1995).

Acknowledgements: Will Featherstone gratefully acknowledges the generous support of the Alexander von Humboldt Stiftung through award number 1120942, as well as the generous hospitality of all staff in the Geodätisches Institut, Universität Stuttgart, Germany. Will Featherstone and Sten Claessens also gratefully acknowledge funding from the Australian Research Council (ARC) through discovery project grant DP0663020. Finally, thanks are extended to the editor and three anonymous reviewers of this manuscript. This is The Institute for Geoscience Research publication number??

NOTE: Since Will Featherstone was Editor in Chief of this Journal at the time of submission, the review process was handled, in confidence, by other Editorial Board members. This has always been standard practice.

\section{References}

1. Bölling K, Grafarend EW (2005) Ellipsoidal spectral properties of the Earth's gravitational potential and its first and second derivatives. J Geod 79(6-7):300-330, DOI: 10.1007/s00190-005-0465-y

2. Claessens SJ (2005) New relations among associated Legendre functions and spherical harmonics. J Geod 79(6-7):398-406, DOI: 10.1007/s00190-005-0483-9

3. Claessens SJ, Featherstone WE (2005) Computation of geopotential coefficients from gravity anomalies on the ellipsoid, in: A Window on the Future of Geodesy, Sansò F (ed.), IAG Symposia Series 128, Springer, Berlin Heidelberg New York, pp 459-464

4. Claessens SJ (2006) Solutions to ellipsoidal boundary value problems for gravity field modelling, $P h D$ Thesis, Department of Spatial Sciences, Curtin University of Technology, Perth, Australia

5. Cruz JY (1986) Ellipsoidal corrections to potential coefficients obtained from gravity anomaly data on the ellipsoid, Rep 371, Dept of Geodetic Sci and Surv, Ohio State Univ, Columbus

6. Grafarend EW (2001) The spherical horizontal and spherical vertical boundary value problem - vertical deflections and geoidal undulations - the completed Meissl diagram. J Geod 75(7-8):363-390, DOI: $10.1007 / \mathrm{s} 001900100186$

7. Heiskanen W, Moritz H (1967) Physical Geodesy. WH Freeman \& Co, San Francisco

8. Hobson EW (1931) The Theory of Spherical and Ellipsoidal Harmonics. Cambridge University Press, Cambridge

9. Holmes SA, Featherstone WE (2002) A unified approach to the Clenshaw summation and the recursive computation of very high degree and order normalised associated Legendre functions. J Geod 76(5):279-299, DOI: 10.1007/s00190-002-0216-2

10. Jekeli C (1981) The downward continuation to the Earth's surface of truncated spherical and ellipsoidal harmonic series of the gravity and height anomalies. Rep 323, Dept of Geodetic Sci and Surv, Ohio State Univ, Columbus

11. Jekeli C (1988) The exact transformation between ellipsoidal and spherical harmonic expansions. manuscr geod 13(2):106-113

12. Jekeli C, Lee J, Kwon J (2007) On the computation and approximation of ultra-high-degree spherical harmonic series. J Geod 81(9):603-615, DOI: 10.1007/s00190-006-0123-z

13. Keller W (2002) Geodetic pseudodifferential operator and the Meissl scheme, in: Geodesy - the Challenge of the Third Millennium, Grafarend EW, Krumm FW and Schwarze VS (eds.), Springer, Berlin Heidelberg New York, pp 206-212

14. Kuhn M, Featherstone WE (2005) On the optimal spatial resolution of crustal mass distributions for forward gravity modelling, in: Proceedings of the 3rd Meeting of the International Gravity and 
Geoid Commission: Gravity and Geoid 2002, Tziavos IN (ed.), Ziti Editions, Thessaloniki, pp 189-194

15. Meissl $P$ (1971) On the linearization of the geodetic boundary value problem, Rep 151, Dept of Geodetic Sci and Surv, Ohio State Univ, Columbus

16. Moritz H (1980a) Advanced Physical Geodesy. Wichmann, Karlsruhe

17. Moritz H (1980b) Geodetic Reference System 1980, Bull Géod 54(4):395-405

18. Pavlis NK, Factor JK, Holmes SA (2007) Terrain-related gravimetric quantities computed for the next EGM, in: Proceedings of the 1st International Symposium of the International Gravity Field Service: Gravity Field of the Earth, Kiliçoğlu A, Forsberg R (eds.), General Command of Mapping, Ankara, pp 318-323

19. Petrovskaya MS, Vershkov AN, Pavlis NK (2001) New analytical and numerical approaches for geopotential modelling. J Geod 75(12): 661-672, DOI: 10.1007/s001900100215

20. Protter MH, Morrey CB (1964) Modern Mathematical Analysis. Addison-Wesley, Reading

21. Rózsa S, Tóth G (2005) Prediction of vertical gravity gradients using gravity and elevation data, in: A Window on the Future of Geodesy, Sansò F (ed.), IAG Symposia Series 128, Springer, Berlin Heidelberg New York, pp 344-349

22. Rummel R, Van Gelderen M (1992) Spectral analysis of the full gravity tensor. Geophys J Int 111(1):159-169

23. Rummel R, Van Gelderen M (1995) Meissl scheme - spectral characteristics of physical geodesy. manuscr geod 20(5):379-385

24. Sjöberg LE (2003) The ellipsoidal corrections to order $e^{2}$ of geopotential coefficients and Stokes' formula. J Geod 77(3-4):139147, DOI: 10.1007/s001900-003-0321-x

25. Sona $G$ (1995) Numerical problems in the computation of ellipsoidal harmonics. J Geod 70(1-2):117-126, DOI: 10.1007/BF00863423

26. Strang G (1986) Linear Algebra and its Applications (3rd edition). Harcourt Brace, Fort Worth

27. Wittwer T, Klees R, Seitz K, Heck B (in press) Ultra-high degree spherical harmonic analysis and synthesis using extended-range arithmetic, J Geod, DOI 10.1007/s00190-007-0172-y 